\title{
Coupled-cavity resonant passive mode-locked Nd:yttrium lithium fluoride laser
}

\author{
U. Keller and T. K. Woodward \\ ATET Bell Laboratories, Holmdel, New Jersey 07733 \\ D. L. Sivco and A. Y. Cho \\ AT\&T Bell Laboratories, Murray Hill, New Jersey 07974
}

Received September 24, 1990; accepted January 7, 1991

\begin{abstract}
We report coupled-cavity resonant passive mode locking of a Nd:YLF laser. This technique has produced 4-ps pulses at a wavelength of $1.047 \mu \mathrm{m}$ with $390-\mathrm{mW}$ average power at a $250-\mathrm{MHz}$ repetition rate, corresponding to a 1.6nJ pulse energy. The Nd:YLF rod was pumped with $1.5 \mathrm{~W}$ of power at $798 \mathrm{~nm}$ from a Ti:sapphire laser. The nonlinear reflector used in the coupled cavity was an InGaAs/GaAs strained layer multiple-quantum-well sample.
\end{abstract}

We introduce a new technique for passive mode locking for a cw pumped Nd:YLF laser, using an InGaAs/ GaAs quantum-well reflector as a nonlinearity in an external coupled cavity. This mode-locking technique is called resonant passive mode locking (RPM) because of the resonant nonlinearity in the coupled cavity. The first demonstration of this novel method was with a Ti:sapphire laser and an $\mathrm{AlGaAs} / \mathrm{GaAs}$ multiple-quantum-well (MQW) reflector. ${ }^{1,2} \mathrm{RPM}$ is markedly different from coupled-cavity mode-locking techniques with a nonresonant nonlinearity (i.e., Kerr type) in the extended cavity and does not need any active cavity-length control for stable pulse trains. ${ }^{2}$ It promises to become a simple and effective way for passive mode locking of laser materials with a high saturation fluence such as Ti:sapphire and $\mathrm{Nd}^{3+}$. doped crystals (YAG, YLF, BEL) and glasses.

An early example of coupled-cavity passive mode locking was the soliton laser, ${ }^{3}$ for which the nonlinear external cavity medium, an optical fiber, supports soliton pulse formation in a net negative group-velocity dispersion regime. This technique has been extended to an external nonlinear coupled cavity with positive group-velocity dispersion, now referred to as additivepulse mode locking ${ }^{4,5}$ (APM) or coupled-cavity mode locking. ${ }^{6}$ The pulse formation is based on the coherent superposition of a pulse in the main cavity and a pulse from the coupled cavity that have experienced a nonlinear phase shift (i.e., self-phase modulation in an optical fiber) leading to constructive interference at the peak of the pulse and destructive interference in the wings. In case of RPM, the coupled cavity has negligible dispersion and the nonlinear quantum-well reflector generates a rapid amplitude modulation that strongly induces mode locking. This fast amplitude nonlinearity has been observed in pump-probe measurements. ${ }^{7}$ A recently developed theory on coupledcavity mode locking with a resonant nonlinearity ${ }^{8}$ suggests that this fast amplitude modulation is enough to mode lock the laser.

This study of the RPM Nd:YLF laser is motivated primarily by the need for practical high-power, highrepetition-rate pulsed laser sources with pulse ener- gies ranging from $1 \mathrm{pJ}$ to $1 \mathrm{~nJ}$, for potential communication and computing applications. At present, highspeed photonic switching elements require switching energies of the order of 1 pJ.,9,10 Diode-pumped mode-locked solid-state lasers offer a practical alternative to mode-locked semiconductor lasers for highpower short-pulse laser sources with clean spatial and spectral mode properties. The output power of active or passive mode-locked semiconductor lasers is typically low, in the maximum range of a few milliwatts, because the laser must be operated near threshold to generate short pulses. Therefore, picojoule-pulse energies are achieved only for repetition rates that are $\leq 1 \mathrm{GHz}$. Amplifier stages are required for picojoule energies at higher repetition rates. For example, with one semiconductor amplifier stage following a modelocked semiconductor laser having a $960-\mathrm{MHz}$ repetition rate and $1.2-\mathrm{mW}$ average output power, $1.3-\mathrm{pJ}$ pulses were amplified to $45 \mathrm{pJ} .{ }^{11}$ In contrast, with high-power diode-laser pump arrays, solid-state lasers can easily supply high power levels, and most difficulties center around mode-locking technqiues. Both active and passive methods have been used. Active mode locking of a diode-pumped Nd:YLF laser at a 2$\mathrm{GHz}$ repetition rate with 7 -ps pulses and $\approx 70$-pJ pulse energy ${ }^{12}$ and of a diode-pumped Nd:YAG laser at a 1$\mathrm{GHz}$ repetition rate with 60 -pJ pulse energy ${ }^{13}$ has been demonstrated. Passive mode locking is generally preferable, as it offers potential for increased repetition rate, greater ease of use, and economy. The passive APM technique applied to a Nd:YAG laser produced 1.7-ps pulses with $25-\mathrm{mW}$ average power when diode pumping was used, ${ }^{14,15}$ and 9 ps with $1.3-\mathrm{W}$ average output power when flash-lamp pumping was used. ${ }^{16}$ An APM Nd:YLF laser produced 2-ps pulses with $10-\mathrm{mW}$ average power with diode pumping ${ }^{14}$ and 3.7-ps pulses with 7-IV average output power with flash-lamp pumping. ${ }^{17}$ A krypton-laser-pumped APM Nd:glass laser produced 380 -fs pulses. ${ }^{18}$ The fiber length (a critical parameter in an APM laser) used in these APM's was $\sim 90 \mathrm{~cm}$, limiting the compactness of this mode-locking scheme. In the case of the RPM Nd:YLF both the crystal and the quantum- 


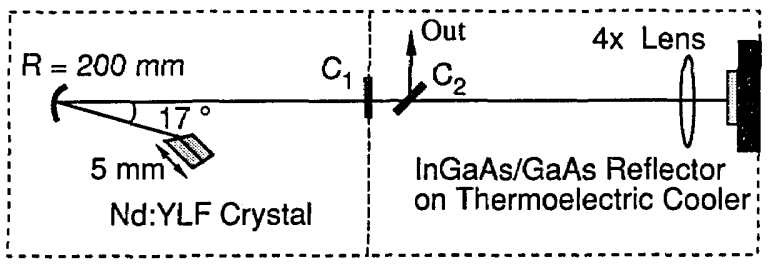

Main Cavity Coupled Cavity

Fig. 1. Cavity design.

well saturable absorber are small, so the laser should be scalable to high repetition rates.

The RPM Nd:YLF laser (Fig. 1) consists of a linear folded $\mathrm{cw} \mathrm{Nd}$ :YLF cavity with a $3 \%$ output coupler, $\mathrm{C}_{1}$, which is coupled to an external low $Q$ cavity with a nonlinear InGaAs/GaAs quantum-well end reflector with a band gap near the lasing wavelength of 1.047 $\mu \mathrm{m} .{ }^{19}$ A tunable output coupler consisting of a halfwave plate and a polarizing beam splitter inside the external cavity is used as the overall output coupler, $\mathrm{C}_{2}$. The Nd:YLI' cavity is end pumped with a Ti:sapphire laser at $798 \mathrm{~nm}$. This 5-mm Nd:YLF rod has its flat end high-reflection coated for the laser wavelength at $1.047 \mu \mathrm{m}$ and antireflection coated for the laser pump at $798 \mathrm{~nm}$, while the other end is cut at Brewster's angle. The linear folded cavity design with an angle of $17^{\circ}$ corrects for the astigmatism arising from the $200-\mathrm{mm}$ radius of curvature of the turning mirror. ${ }^{20}$ Output coupler $\mathrm{C}_{1}$ is slightly wedged to minimize unwanted étalon effects in the cavity. The nominal $\mathrm{Nd}^{3+}$ concentration is $1.5 \%$. The calculated cavity beam waist in the Nd:YLF rod is elliptical, with a beam radius of $66 \mu \mathrm{m}$ in the saggital plane and $93 \mu \mathrm{m}$ in the tangential plane. To produce a $\mathrm{TEM}_{00}$ mode, the Ti:sapphire pump laser beam is focused to a smaller spot size of $\approx 46-\mu \mathrm{m}$ radius by an $88-\mathrm{mm}$ focusing lens. The Nd:YLF laser cavity with its $3 \%$ output coupler forms a stable cw laser with a slope efficiency of $57 \%$ and a pump threshold of $\approx 48 \mathrm{~mW}$. This high slope efficiency and low threshold are possible because of the small pump laser area. The mode-beating frequency (i.e., the cavity repetition rate) of the $\mathrm{cw}$ Nd:YLF laser is $\approx 250 \mathrm{MHz}$. We used a microscope lens with a focusing length of $30.6 \mathrm{~mm}$ to focus the beam to an estimated $\approx 30-\mu \mathrm{m}$ diameter spot onto the nonlinear MQW reflector that forms the end mirror of the coupled cavity. The nonlinear quantum-well reflector is an $\operatorname{In}_{x} \mathrm{Ga}_{1-x} \mathrm{As} / \mathrm{GaAs} \mathrm{MQW}$ optical modulator with a band gap near $1.047 \mu \mathrm{m}$, described in more detail in Ref. 19 as sample D. Because there is no dielectric mirror grown underneath the MWQ structure and the $\mathrm{GaAs}$ substrate is transmissive at 1.047 $\mu \mathrm{m}$, we evaporated a gold mirror onto a $\mathrm{MQW}$ layer consisting of $\approx 5 \mathrm{~nm}$ of chrome as the sticking layer and $\approx 250 \mathrm{~nm}$ of gold. The reflector is indium bonded onto a copper substrate, which is mounted onto a thermoelectric cooler.

The pulse width is measured with a noncollinear autocorrelator (Fig. 2, solid curve) and compared with an ideal 4.0-ps FWHM sech ${ }^{2}$ pulse (Fig. 2, dashed curve). The Nd:YLF rod is cw pumped with $1.5 \mathrm{~W}$ of power, generating $390 \mathrm{~mW}$ of average output power $P_{1}$ at an $\approx 250-\mathrm{MHz}$ repetition rate, corresponding to 1.6 $\mathrm{nJ}$ pulse energy with an $\approx 48 \%$ overall output coupler $\mathrm{C}_{2}$. The pulses are simultaneously monitored with a noncollinear autocorrelator, a sampling scope, a rf spectrum analyzer, and an optical spectrum analzyer. The pulse on the sampling scope is well defined, and its width is limited by the overall time resolution of photodiode and sampling head to $\approx 50$-ps pulse duration. The stability of the mode-locked pulses is not so good as for the RPM Ti:sapphire laser. ${ }^{2}$ We observe as much as $30 \%$ amplitude fluctuations on the autocorrelation trace. The pulse width remains constant. The average power reflected from the quantum-well absorber and injected back into the main cavity is only $3 \%$ because of significant diffraction from surface striations on the quantum-well reflector. ${ }^{19}$ This diffraction also contributes to the poor mode quality of the reflected beam. The poor reflection from the quantum-well sample may also be the reason for the excess wings in the autocorrelation trace (Fig. 2).

The 4-ps pulses are achieved at a slightly detuned cavity length of $-0.22 \mathrm{~mm}$. When the two cavities are exactly matched, the laser is extremely unstable, with no defined pulses on the sampling scope, which is consistent with the theoretical model ${ }^{8}$ Mode locking in this regime would require active cavity-length control. Increasing the cavity-length detuning increases the pulse width, and at more than $\approx 0.9-\mathrm{mm}$ detuning the autocorrelation traces begin to break up into multiple $\approx 4$-ps pulses superimposed upon a longer pulse. These pulses are separated by the cavity-length detuning time. At even larger detunings these short pulses do not overlap anymore, and they show a $2: 1$ intensity ratio of peak to background, which implies that they are coherence spikes. Therefore at larger cavity detunings the laser is not properly mode locked. We believe that the detuning range over which mode locking is observed can be improved with better reflector quality, as discussed above.

When we vary the output coupler $C_{1}$ of the cw Nd:YLF laser, the mode-locking performance is improved as the output coupler $\mathrm{C}_{1}$ is changed from $10 \%$ to $5 \%$ and then to $3 \%$. For an output coupler of $10 \%$ the nonlinear coupled cavity produces strong noise sidebands up to $\approx 10 \mathrm{~dB}$ below the first laser harmonic

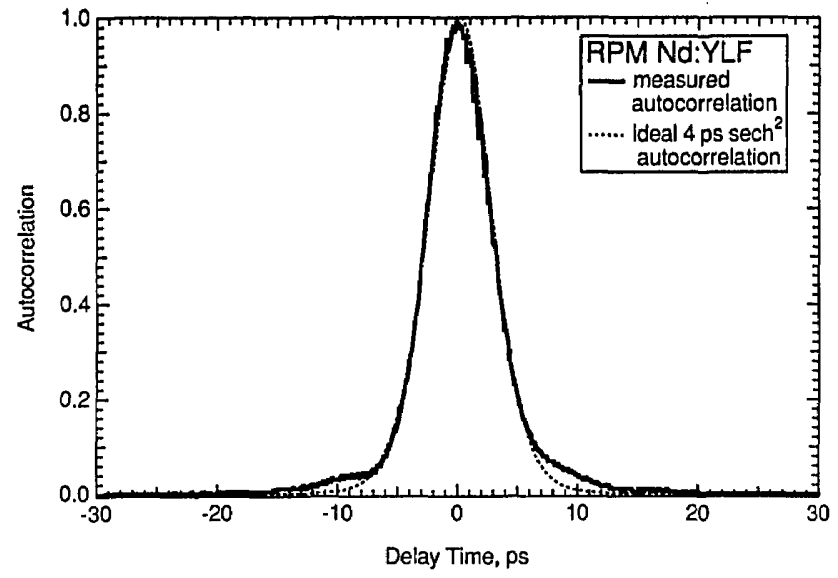

Fig. 2. 4-ps autocorrelation trace assuming a $\operatorname{sech}^{2}$ pulse shape. 


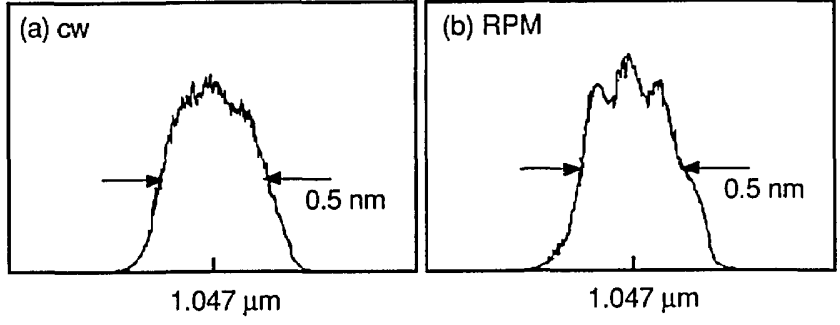

Fig. 3. Time-averaged optical spectrum under (a) cw operation and (b) mode-locked operation.

owing to enhanced relaxation oscillations of the Nd:YLF cavity. Decreasing the output coupler decreases these noise sidebands, which are greatly mitigated with the $3 \%$ output coupler. We observe optimized performance with an overall output coupler $\mathrm{C}_{2}$ (Fig. 1) in the range of $50 \%$ to $60 \%$ at the abovementioned power levels.

Monitoring the output of the laser with the rf spectrum analyzer, we observe a well-defined first laser harmonic near $250 \mathrm{MHz}$ that is $\approx 60 \mathrm{~dB}$ above the mode beating signal from the $\mathrm{cw}$ Nd:YLF laser with the external cavity blocked. By shortening the external cavity we increase the repetition rate. This pulling effect is larger with smaller output couplers $\mathrm{C}_{2}$ in the coupled cavity. Typically, with a $\approx 50 \%$ output coupler $\mathrm{C}_{2}$ and with $0.1-\mathrm{mm}$ cavity detuning we measure a pulse-repetition-rate change in the kilohertz regime. This frequency pulling effect is easy to understand: At a cavity length detuning of $-0.22 \mathrm{~mm}$, the 4-ps pulse in the main cavity is still overlapping the reinjected advanced (by $\approx 1.5 \mathrm{ps}$ ) pulse from the external cavity. The total pulse, which is the sum of the two, will have a slightly advanced timing depending on the amplitude of the reinjected pulse.

The time-averaged optical spectrum for cw operation with an optical spectrum analyzer is shown in Fig. 3(a), and for that mode-locked operation in Fig. 3(b). The time-averaged optical spectrum has a FWHM of $\approx 0.5 \mathrm{~nm}$, which would imply that the 4-ps pulses are $\approx 1.7$ times transform limited. However, in RPM the time-averaged optical spectrum [Fig. 3(b)] includes the self-frequency shifts of the laser to maintain stable pulse trains that do not correspond to the bandwidth of the mode-locked pulses. The optical bandwidth of the mode-locked pulses decreases with increased cavity length detuning, and a more structured time-dependent optical spectrum under the mode-locked condition is consistent with stabilization of coupled-cavity operation by self-frequency adjustments. ${ }^{2,8}$

In summary, we have demonstrated RPM of a Nd:YLF laser and achieved 4-ps pulse duration. This introduces a new technique of mode locking $\mathrm{Nd}^{3+}$. based solid-state lasers. With only $1.5 \mathrm{~W}$ of pump power at $798 \mathrm{~nm}$, we have generated pulses with energies as high as $\approx 1.6 \mathrm{~nJ}$ at a repetition rate of $250 \mathrm{MHz}$. As we have demonstrated with the RPM Ti:sapphire laser, ${ }^{2}$ the pulse energy scales inversely with the repetition rate because the average power remains constant. Here the RPM Nd:YLF laser is pumped by a Ti:sapphire laser. Diode pumping is a straightforward extension. However, owing to the poor spatial and spectral mode properties of high-power diode arrays, the diode pump power cannot be focused so tightly as the Ti:sapphire pump power. Therefore the mode volume in the Nd:YLF crsytal has to be enlarged to guarantee $\mathrm{TEM}_{00}$ mode operation, which will increase the pump threshold. Diode pumping may produce a practical high-power laser source with hundreds of picojoules of pulse energy at repetition rates well above $1 \mathrm{GHz}$ for applications requiring high-energy, high-repetition-rate laser sources such as photonic switching for optical communication and computing.

The authors thank Wayne H. Knox, who inspired the original RPM work on the Ti:sapphire laser on which this work is based, Kurt J. Weingarten of Lightwave Electronics Corporation for the Nd:YLF laser crystal and helpful discussions, and Greg Raybon for the antireflection coating of the quantum-well reflectors.

\section{References}

1. U. Keller, W. H. Knox, and H. Roskos, in Ultrafast Phenomena VII, C. B. Harris, E. P. Ippen, G. A. Mourou, and A. H. Zewail, eds. (Springer-Verlag, Berlin, 1990), p. 69.

2. U. Keller, W. H. Knox, and H. Roskos, Opt. Lett. 15, 1377 (1990).

3. L. F. Mollenauer and R. H. Stolen, Opt. Lett. 9, 13 (1984).

4. C. R. Pollock, in Digest of Conference on Lasers and Electro-Optics (Optical Society of America, Washington, D.C., 1988), paper PD7-1; P. Yakymyshyn, J. F. Pinto, and C. R. Pollock, Opt. Lett. 14, 621 (1989).

5. J. Mark, L. Y. Liu, K. L. Hall, H. A. Haus, and E. P. Ippen, Opt. Lett. 14, 48 (1989).

6. P. N. Kean, X. Zhu, D. W. Crust, R. S. Grant, N. Langford, and W. Sibbett, Opt. Lett. 14, 39 (1989).

7. U. Keller, G. W. 't Hooft, W. H. Knox, and T. K. Woodward, in Digest of Topical Meeting on Optoelectronics (Optical Society of America, Washington, D.C., 1990), paper WB2.

8. H. A. Haus, U. Keller, and W. H. Knox, "Theory of coupled-cavity mode locking with a resonant nonlinearity," J. Opt. Soc. Am. B (to be published).

9. M. N. Islam, C. E. Soccolich, and D. A. B. Miller, Opt. Lett. 15, 909 (1990).

10. D. A. B. Miller, Opt. Quantum Electron. 22, S61 (1990).

11. P. J. Delfyett, C.-H. Lee, G. A. Alphonse, and J. C. Connolly, Appl. Phys. Lett. 57, 971 (1990).

12. K. J. Weingarten, D. C. Shannon, R. W. Wallace, and U. Keller, Opt. Lett. 15, 962 (1990).

13. S. J. Walker, H. Avramopoulos, and T. Sizer II, Opt. Lett. 15, 1070 (1990).

14. J. Goodberlet, J. Jacobson, J. Wang, and P. A. Schulz, in Ultrafast Phenomena VII, C. B. Harris, E. P. Ippen, G. A. Mourou, and A. H. Zewail, eds. (Springer-Verlag, Berlin, 1990), p. 11.

15. J. Goodberlet, J. Jacobson, J. G. Fujimoto, P. A. Schulz, and T. Y. Fan, Opt. Lett. 15, 504 (1990).

16. L. Y. Liu, J. M. Huxley, E. P. Ippen, and H. A. Haus, Opt. Lett. 15, 553 (1990).

17. J. M. Liu and J. K. Chee, Opt. Lett. 15, 685 (1990).

18. F. Krausz, Ch. Spielmann, T. Brabec, E. Wintner, and A. J. Schmidt, Opt. Lett. 15, 1082 (1990).

19. T. K. Woodward, T. Sizer II, D. L. Sivco, and A. Y. Cho, Appl. Phys. Lett. 57, 548 (1990).

20. H. W. Kogelnick, E. P. Ippen, A. Dienes, and C. V. Shank, IEEE J. Quantum Electron. QE-8, 373 (1972). 